\title{
Anisotropic hydrodynamics, holography, and the chiral magnetic effect
}

\author{
Ilmar Gahramanov, Tigran Kalaydzhyan, and Ingo Kirsch \\ DESY Hamburg, Theory Group, Notkestrasse 85, D-22607 Hamburg, Germany, and Zentrum für Mathematische Physik, \\ Universität Hamburg, Bundesstrasse 55, D-20146 Hamburg
}

(Received 29 March 2012; published 26 June 2012)

\begin{abstract}
We discuss a possible dependence of the chiral magnetic effect (CME) on the elliptic flow coefficient $v_{2}$. We first study this in a hydrodynamic model for a static anisotropic plasma with multiple anomalous $U(1)$ currents. In the case of two charges, one axial and one vector, the CME formally appears as a firstorder transport coefficient in the vector current. We compute this transport coefficient and show its dependence on $v_{2}$. We also determine the CME coefficient from first-order corrections to the dual anti-de Sitter background using the fluid-gravity duality. For small anisotropies, we find numerical agreement with the hydrodynamic result.
\end{abstract}

DOI: 10.1103/PhysRevD.85.126013

PACS numbers: 47.75.+f, 11.15.- q, 11.25.Tq, 12.38.Mh

\section{INTRODUCTION}

In the last couple of years the chiral magnetic effect (CME) has attracted much attention as a candidate for the explanation of an experimentally observed charge asymmetry in heavy-ion collisions, as seen by the STAR [1], PHENIX [2] and ALICE [3] collaborations. The CME is a hypothetical phenomenon which states that, in the presence of a magnetic field $\vec{B}$, an electric current is generated along $\vec{B}$ in the background of topologically nontrivial gluon fields [4,5]. Analogous effects were found earlier in neutrino [6], electroweak [7] and condensed matter physics [8]. Lattice QCD results [9-11] suggest the existence of the effect, although the magnitude of the CMEinduced charge asymmetry may be too small to explain the observed charge asymmetry [12].

In a recent experiment, the charge separation is measured as a function of the elliptic flow coefficient $v_{2}$ [13]. The data is taken from (rare) $\mathrm{Au}+\mathrm{Au}$ collisions with 20-40\% centrality but different $v_{2}$. In this way $v_{2}$ is varied while at the same time the number of participating nucleons (and therefore the magnetic field) is kept almost constant. The plots in [13] suggest that the charge separation is proportional to $v_{2}$. If this holds true, the charge separation will depend on the event anisotropy.

In this paper we address the question of whether and how the CME depends on the elliptic flow $v_{2}$. We study this both in hydrodynamics and in terms of a holographic gravity dual. The hydrodynamical approach to the CME and CME-related phenomena was proposed in [14-20]. There, the CME appears in form of a nonvanishing transport coefficient in the electric current, $\vec{j}=\kappa_{B} \vec{B}$, which measures the response of the system to an external magnetic field [14,21]. In [20], the chiral magnetic conductivity in an isotropic fluid was determined as

$$
\kappa_{B}=C \mu_{5}\left(1-\frac{\mu \rho}{\epsilon+P}\right) .
$$

The first term is the standard term for the CME and depends only on the axial anomaly coefficient $C$ and the axial chemical potential $\mu_{5}$. The second term proportional to the factor $\frac{\rho}{\epsilon+P}$ depends on the dynamics of the fluid and has a chance to depend on $v_{2}$ in the anisotropic case.

In the first part of the paper we study this in a hydrodynamic model for an anisotropic fluid with multiple anomalous $U(1)$ charges (This model extends those in [22-24]). We compute the CME coefficient $\kappa_{B}$ and express the result in terms of the momentum anisotropy $\varepsilon_{p}$ [25] defined as

$$
\varepsilon_{p}=\frac{\left\langle P_{T}-P_{L}\right\rangle}{\left\langle P_{T}+P_{L}\right\rangle}
$$

where $P_{T}$ and $P_{L}$ are the pressures in the plane transverse to the beam line (In our conventions the indices $L$ and $T$ refer to the longitudinal and transverse direction with respect to an anisotropy vector $v_{\mu}$ normal to the reaction plane, see Fig. 1). A sketch of $\varepsilon_{p}$ as a function of the proper time $\tau$ is shown in Fig. 1. $\varepsilon_{p}$ describes the buildup of the elliptic flow in off-central collisions. Our model describes a state after thermalization with unequal pressures $P_{T} \neq P_{L}$. At freeze-out $\varepsilon_{p}$ roughly equals $v_{2}$, and we find that for small anisotropies the CME coefficient $\kappa_{B}$ increases linearly with $v_{2}$.

In the second part of the paper we perform a holographic computation of $\kappa_{B}$ in the dual gravity model. A similar computation was previously done in [20] for the STU model [26], a string-theory-inspired prototype of an (isotropic) anti-de Sitter (AdS) black hole solution with three $U(1)$ charges. Other holographic approaches to the CME can be found in [27-37].

In the anisotropic case, we first need to construct an appropriate gravity background. As an ansatz, we choose a multiply charged AdS black hole solution with some additional functions $w_{L}$ and $w_{T}$ inserted which will make the background anisotropic and $\varepsilon_{p}$-dependent. Since 


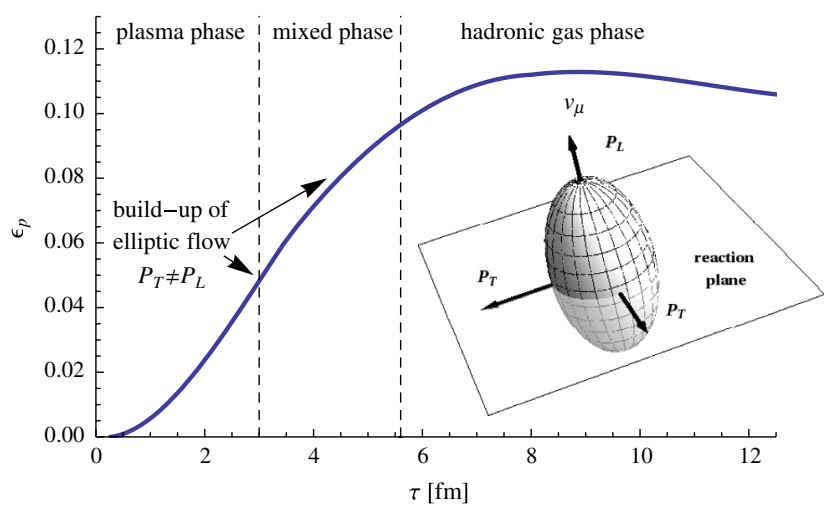

FIG. 1 (color online). Sketch of the time evolution of the momentum anisotropy $\varepsilon_{p}$ (based on [53]). The small figure shows the orientation of $P_{L}$ and $P_{T}$ with respect to the reaction plane.

analytical solutions for charged anisotropic backgrounds are notoriously difficult to find, we will use shooting techniques to find a numerical solution. Other AdS backgrounds dual to anisotropic fluids are constructed in [38-40].

As the AdS solution in [38], the background is static and does not describe the process of isotropization. Even though such models have some limitations [38], they are nevertheless useful for the computation of transport coefficients. We show this, following [20], by determining $\kappa_{B}$ from the first-order corrections to this background using the fluid-gravity duality [41]. For small anisotropies, we find numerical agreement with the hydrodynamic result for $\kappa_{B}$. Other (dissipative) transport coefficients in strongly coupled anisotropic plasmas are discussed in [42-44].

The paper is organized as follows. In Sec. II we review the hydrodynamics of an anisotropic relativistic fluid with several $U(1)$ charges and triangle anomalies. We then compute the vortical and magnetic conductivities of such a fluid by extending the method of Son and Surowka [14] to the anisotropic case. In Sec. III we construct the dual gravity background and present a numerical solution for its gauge field and metric functions. In Sec. IV we use this background to perform a holographic computation of the vortical and magnetic conductivities.

\section{HYDRODYNAMICS OF ANISOTROPIC FLUIDS WITH TRIANGLE ANOMALIES}

The hydrodynamic regime of isotropic relativistic fluids with triangle anomalies has been studied in [14-19], and much can be taken over to the anisotropic case. Such fluids typically contain $n$ anomalous $U(1)$ charges which commute with each other. The anomaly coefficients are given by a totally symmetric rank-3 tensor $C^{a b c}$. The hydrodynamic equations are

$$
\partial_{\mu} T^{\mu \nu}=F^{a \nu \lambda} j_{\lambda}^{a}, \quad \partial_{\mu} j^{a \mu}=C^{a b c} E^{b} \cdot B^{c},
$$

where $\quad E^{a \mu}=F^{a \mu \nu} u_{\nu}, \quad B^{a \mu}=\frac{1}{2} \epsilon^{\mu \nu \alpha \beta} u_{\nu} F_{\alpha \beta}^{a} \quad(a=$ $1, \ldots, n)$ are electric and magnetic fields, and $F_{\mu \nu}^{a}=$ $\partial_{\mu} A_{\nu}^{a}-\partial_{\nu} A_{\mu}^{a}$ denotes the gauge field strengths. As in [14], we expand the constitutive equations for $T^{\mu \nu}$ and $j^{\mu}$ up to first order, taking $A_{\mu}^{a} \sim O\left(p^{0}\right)$ and $F_{\mu \nu}^{a} \sim O(p)$. The gauge fields $A_{\mu}^{a}$ are nondynamical.

In anisotropic relativistic fluids, the hydrodynamic equations are again given by (3) but the stress-energy tensor $T^{\mu \nu}$ and $U(1)$ currents $j^{a \mu}$ now have the more general form ${ }^{1}$

$$
\begin{gathered}
T^{\mu \nu}=\left(\epsilon+P_{T}\right) u^{\mu} u^{\nu}+P_{T} g^{\mu \nu}-\Delta v^{\mu} v^{\nu}+\tau^{\mu \nu}, \\
j^{a \mu}=\rho^{a} u^{\mu}+\nu^{a \mu},
\end{gathered}
$$

where $\epsilon$ is the energy density, $\rho^{a}$ are the $U(1)$ charge densities, $\Delta=P_{T}-P_{L}$, and $P_{T}$ and $P_{L}$ denote the transverse and longitudinal pressures, respectively [22-24]. $g_{\mu \nu}$ is the metric with signature $(-,+,+,+) . \tau^{\mu \nu}$ and $\nu^{a \mu}$ denote higher-gradient corrections, for which we require $u_{\mu} \tau^{\mu \nu}=0$ and $u_{\mu} \nu^{a \mu}=0$.

The four-vectors $u^{\mu}$ and $v^{\mu}$ describe the flow of the fluid and the direction of the longitudinal axis, respectively. The vector $v^{\mu}$ is spacelike and orthogonal to $u^{\mu}$,

$$
u_{\mu} u^{\mu}=-1, \quad v_{\mu} v^{\mu}=1, \quad u_{\mu} v^{\mu}=0 .
$$

It is convenient to define the proper time $\tau$ by $\partial^{\nu} \ln \tau \equiv$ $v^{\mu} \partial_{\mu} v^{\nu}$ [23]. In the rest frame of the fluid, $u^{\mu}=$ $(1,0,0,0)$ and $v^{\mu}=(0,0,0,1)$, the stress-energy tensor becomes diagonal,

$$
T^{\mu \nu}=\left(\begin{array}{cccc}
\epsilon & 0 & 0 & 0 \\
0 & P_{T} & 0 & 0 \\
0 & 0 & P_{T} & 0 \\
0 & 0 & 0 & P_{L}
\end{array}\right) .
$$

In conformal fluids, the stress-energy tensor is traceless, $T_{\mu}^{\mu}=0$, and $\epsilon=2 P_{T}+P_{L}$. Clearly, the isotropic case corresponds to equal pressures $P_{T}$ and $P_{L}, P=P_{T}=P_{L}$.

For simplicity, we restrict to the case of a single charge in Secs. II A and II B, $n=1$. In Secs. II C and II D we generalize our findings to arbitrary $n$ and discuss the case $n=2$, which is relevant for the CME.

\section{A. Thermodynamics of an anisotropic fluid with chemical potential $(n=1)$}

Hydrodynamic models for an anisotropic fluid (without chemical potential) have been studied in [22-24]. Following these works, we derive some thermodynamic identities, now for the case of a fluid with a chemical potential $\mu$.

\footnotetext{
${ }^{1}$ The symmetries allow in principle for more general currents $j^{a \mu}=\rho^{a} u^{\mu}+c^{a} v^{\mu}+\nu^{a \mu}$ with some coefficients $c^{a}$. Here we switch off all the "electric" background currents, $c^{a}=0$.
} 
These identities can be found by computing the quantity $I_{0}=u_{\nu} \partial_{\mu} T^{\mu \nu}+\mu \partial_{\mu} j^{\mu}$ at zeroth order. Since the righthand side of (3) can be dropped at order $O\left(p^{0}\right)$, we have $I_{0}=0$. Using $\partial_{\mu}\left(s u^{\mu}\right)=0$, we get

$$
\begin{gathered}
u_{\nu} \partial_{\mu} T^{\mu \nu}=-u^{\mu} \partial_{\mu} \epsilon-\left(\epsilon+P_{T}\right) \partial_{\mu} u^{\mu}-\Delta u_{\nu} \partial^{\nu} \ln \tau \\
=-u^{\mu} \partial_{\mu} \epsilon+\frac{\epsilon+P_{T}}{s} u^{\mu} \partial_{\mu} s-\frac{\Delta}{\tau} u^{\mu} \partial_{\mu} \tau, \\
\mu \partial_{\mu} j^{\mu}=\mu\left(\partial_{\mu} \rho\right) u^{\mu}-\frac{\mu \rho}{s} u^{\mu} \partial_{\mu} s .
\end{gathered}
$$

As in [23], we consider a generalized energy density $\epsilon=\epsilon(s, \rho, \tau)$, which depends not only on the entropy density $s$ and particle density $\rho$ but also on the new variable $\tau$. Its differential is

$$
d \epsilon=\left(\frac{\partial \epsilon}{\partial s}\right)_{\rho, \tau} d s+\left(\frac{\partial \epsilon}{\partial \rho}\right)_{s, \tau} d \rho+\left(\frac{\partial \epsilon}{\partial \tau}\right)_{s, \rho} d \tau,
$$

with

$$
\left(\frac{\partial \epsilon}{\partial s}\right)_{\rho, \tau}=T, \quad\left(\frac{\partial \epsilon}{\partial \rho}\right)_{s, \tau}=\mu, \quad\left(\frac{\partial \epsilon}{\partial \tau}\right)_{s, \rho}=-\frac{\Delta}{\tau} .
$$

The temperature and the chemical potential are defined in the usual way. If we also impose $(\partial \epsilon / \partial \tau)_{s, \rho}=-\Delta / \tau$ and substitute (10) into (8), then $I_{0}=0$ implies the following thermodynamical identities for an anisotropic fluid:

$$
\begin{gathered}
\epsilon+P_{T}=T s+\mu \rho, \\
d P_{T}=\frac{\Delta}{\tau} d \tau+s d T+\rho d \mu, \\
d \epsilon=T d s+\mu d \rho-\frac{\Delta}{\tau} d \tau,
\end{gathered}
$$

in agreement with [23] for $\mu=0$.

\section{B. Vortical and magnetic coefficients $(n=1)$}

We now discuss corrections to the $U(1)$ current $j^{\mu} \equiv j^{1 \mu}(n=1)$. In anisotropic fluids the transport coefficients are usually promoted to tensors such that one should consider first-derivative corrections of the type

$$
\nu^{\mu}=\left(\xi_{\omega}\right)_{\nu}^{\mu} \omega^{\nu}+\left(\xi_{B}\right)^{\mu}{ }_{\nu} B^{\nu},
$$

where $\omega^{\mu}=\frac{1}{2} \epsilon^{\nu \rho \sigma \mu} u_{\nu} \partial_{\rho} u_{\sigma}$ is the vorticity, and $B^{\mu}$ is an external magnetic field. In Landau frame $u_{\mu} \nu^{\mu}=0$ and therefore $u_{\mu}\left(\xi_{\omega}\right)_{\nu}^{\mu} \omega^{\nu}=0$ (and similar for $\left(\xi_{B}\right)^{\mu}{ }_{\nu}$ ). This is satisfied e.g. for $\left(\xi_{\omega}\right)^{\mu}{ }_{\nu}=\xi_{\omega} \delta^{\mu}{ }_{\nu}$, since $u_{\mu} \omega^{\mu}=0$ (We do not consider other components of $\xi_{\omega}$ here). We therefore restrict to consider corrections of the type

$$
\nu^{\mu}=\xi_{\omega} \omega^{\mu}+\xi_{B} B^{\mu},
$$

as in the isotropic case [14]. Our goal is to compute the vortical and magnetic conductivities $\xi_{\omega}$ and $\xi_{B}$. These transport coefficients can be found by assuming the existence of an entropy current $s^{\mu}$ with a non-negative derivative, $\partial_{\mu} s^{\mu} \geq 0$. The computation closely follows that of [14].

The hydrodynamic Eqs. (3) imply that the quantity

$$
I_{1}=u_{\nu} \partial_{\mu} T^{\mu \nu}+\mu \partial_{\mu} j^{\mu}+E^{\mu} \nu_{\mu}-\mu C E^{\mu} B_{\mu}
$$

vanishes at first order, $I_{1}=0$. Substituting the explicit expressions for the stress-energy tensor and $U(1)$ currents into $I_{1}$ and using the thermodynamical identities (12) and (14), we find

$$
\begin{aligned}
\partial_{\mu}\left(s u^{\mu}-\frac{\mu}{T} \nu^{\mu}\right)= & -\frac{1}{T} \partial_{\mu} u_{\nu} \tau^{\mu \nu}-\nu^{\mu}\left(\partial_{\mu} \frac{\mu}{T}-\frac{E_{\mu}}{T}\right) \\
& -C \frac{\mu}{T} E \cdot B,
\end{aligned}
$$

which is exactly the same equation for the entropy production as in the isotropic case [14].

In the following, we will need the identities

$$
\begin{aligned}
& \partial_{\mu} \omega^{\mu}=-\frac{2}{\epsilon+P_{T}} \omega^{\mu}\left(\partial_{\mu} P_{T}-\Delta \partial_{\mu} \ln \tau-\rho E_{\mu}\right), \\
& \partial_{\mu} B^{\mu}=-2 \omega^{\mu} E_{\mu}-\frac{B^{\mu}}{\epsilon+P_{T}}\left(\partial_{\mu} P_{T}-\Delta \partial_{\mu} \ln \tau-\rho E_{\mu}\right),
\end{aligned}
$$

which we derived from ideal hydrodynamics in Appendix A. In deriving these identities we assumed that the fluid satisfies

$$
\partial_{\mu} v^{\mu}=0, \quad v^{\mu} \partial_{\mu} \Delta=0 .
$$

The first equation is basically a "continuity equation" for the vector $v^{\mu}$. There are no sources for the generation of anisotropy. The second equation imposes an orthogonality relation between the gradient of the pressure difference $\Delta=P_{T}-P_{L}$ and $v^{\mu}$.

As in [14], we assume a generalized entropy current of the form

$$
s^{\mu}=s u^{\mu}-\frac{\mu}{T} \nu^{\mu}+D \omega^{\mu}+D_{B} B^{\mu},
$$

where $\xi_{\omega}, \xi_{B}, D$, and $D_{B}$ are functions of $T, \mu$ and $\tau$. We now compute $\partial_{\mu} s^{\mu}$, using (18) and (19) and impose $\partial_{\mu} s^{\mu} \geq 0$. Since the coefficients in front of $\omega^{\mu}, B^{\mu}$, $\omega_{\mu} E^{\mu}$ and $E_{\mu} B^{\mu}$ inside $\partial_{\mu} s^{\mu}$ can have either sign, we require them to vanish and obtain the following four differential equations:

$\partial_{\mu} D-\frac{2 D}{\epsilon+P_{T}}\left(\partial_{\mu} P_{T}-\Delta \partial_{\mu} \ln \tau\right)-\xi_{\omega} \partial_{\mu} \frac{\mu}{T}=0$,

$\partial_{\mu} D_{B}-\frac{D_{B}}{\epsilon+P_{T}}\left(\partial_{\mu} P_{T}-\Delta \partial_{\mu} \ln \tau\right)-\xi_{B} \partial_{\mu} \frac{\mu}{T}=0$, 


$$
\begin{gathered}
\frac{2 \rho D}{\epsilon+P_{T}}-2 D_{B}+\frac{\xi_{\omega}}{T}=0, \\
\frac{\rho D_{B}}{\epsilon+P_{T}}+\frac{\xi_{B}}{T}-C \frac{\mu}{T}=0 .
\end{gathered}
$$

For $\Delta=0$, these equations reduce to those in the isotropic case [14].

In Appendix B we solve (22)-(25) for $D, D_{B}, \xi_{\omega}$ and $\xi_{B}$. As a result, we find the vortical and magnetic conductivities

$$
\begin{aligned}
& \xi_{\omega}=C\left(\mu^{2}-\frac{2}{3} \frac{\rho \mu^{3}}{\epsilon+P_{T}}\right)+\mathcal{O}\left(T^{2}\right), \\
& \xi_{B}=C\left(\mu-\frac{1}{2} \frac{\rho \mu^{2}}{\epsilon+P_{T}}\right)+\mathcal{O}\left(T^{2}\right),
\end{aligned}
$$

where $\mathcal{O}\left(T^{2}\right)$ denotes terms proportional to $T^{2}$, see (B14) in Appendix B. These terms are related to gravitational triangle anomalies $[18,45]$ and may, in the anisotropic case, depend on the proper time $\tau$. In the absence of gravitational anomalies, which we do not discuss in this paper, the conductivities do not depend on $\tau$. Apart from these changes in $\mathcal{O}\left(T^{2}\right)$, the relations have the same form as in the isotropic case but with $P$ replaced by the transverse pressure $P_{T}$.

\section{Multiple charge case ( $n$ arbitrary)}

The generalization of the previous computation to a fluid with multiple anomalous $U(1)$ charges is straightforward, and we only state the result here. The corrections $\nu^{a \mu}$ of the currents $j^{a \mu}$ in (5) are

$$
\nu^{a \mu}=\xi_{\omega}^{a} \omega^{\mu}+\xi_{B}^{a b} B^{b \mu},
$$

with [terms of order $\mathcal{O}\left(T^{2}\right)$ ignored]

$$
\begin{gathered}
\xi_{\omega}^{a}=C^{a b c} \mu^{b} \mu^{c}-\frac{2}{3} \rho^{a} C^{b c d} \frac{\mu^{b} \mu^{c} \mu^{d}}{\epsilon+P_{T}}, \\
\xi_{B}^{a b}=C^{a b c} \mu^{c}-\frac{1}{2} \rho^{a} C^{b c d} \frac{\mu^{c} \mu^{d}}{\epsilon+P_{T}} .
\end{gathered}
$$

These are simple generalizations of the corresponding conductivities in the isotropic case [14,18].

\section{Chiral magnetic and vortical effect $(n=2)$}

Physically, the most interesting case is that involving two charges $(n=2)[16,17,20]$. The chiral magnetic effect [4] can be described by one axial and one vector $U(1)$, denoted by $U(1)_{A} \times U(1)_{V}$. A convenient notation for the gauge fields and currents is $(a, b, \ldots=1,2)$

$$
A_{\mu}^{A}=A_{\mu}^{1}, \quad A_{\mu}^{V}=A_{\mu}^{2}, \quad j_{5}^{\mu}=j^{1 \mu}, \quad j^{\mu}=j^{2 \mu} .
$$
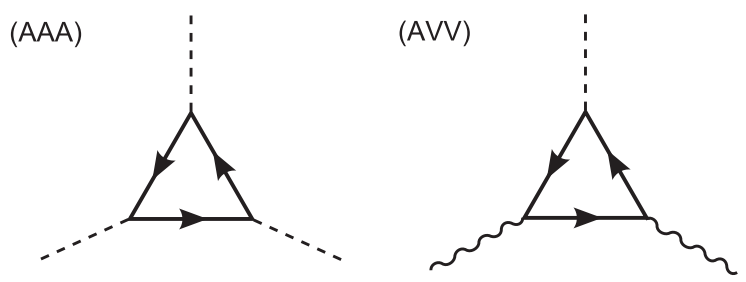

FIG. 2. Anomalous diagrams corresponding to $C^{111}$ (left) and to $C^{122}=C^{221}=C^{212}$ (right). Dashed (wavy) lines denote the axial (vector) currents/fields.

Let us now derive the chiral magnetic and vortical effects from (28) and (29). $C$ - parity allows for two anomalous triangle diagrams, (AAA) and (AVV), shown in Fig. 2, while diagrams of the type (VVV) and (VAA) vanish. Accordingly, the anomaly coefficients are

$$
\begin{aligned}
& C^{121}=C^{211}=C^{112}=0, \\
& C^{222}=0, \quad(\mathrm{VVV}) \\
& C^{111} \neq 0, \quad(\mathrm{AAA}) \\
& C^{122}=C^{221}=C^{212} \neq 0 .
\end{aligned}
$$

The hydrodynamic Eqs. (3) then imply nonconserved vector and axial currents,

$$
\begin{aligned}
& \partial_{\mu} j^{\mu}=-\frac{1}{4}\left(C^{212} F_{\mu \nu}^{A} \tilde{F}^{V \mu \nu}+C^{221} F_{\mu \nu}^{V} \tilde{F}^{A \mu \nu}\right), \\
& \partial_{\mu} j_{5}^{\mu}=-\frac{1}{4}\left(C^{111} F_{\mu \nu}^{A} \tilde{F}^{A \mu \nu}+C^{122} F_{\mu \nu}^{V} \tilde{F}^{V \mu \nu}\right),
\end{aligned}
$$

where we rewrote $E^{b} \cdot B^{c}=-\frac{1}{4} F_{\mu \nu}^{b} \tilde{F}^{c \mu \nu}$ (with $\tilde{F}^{a \mu \nu}=$ $\left.\frac{1}{2} \varepsilon^{\mu \nu \rho \sigma} F_{\rho \sigma}^{a}\right)$.

To restore conservation of the vector current, we add the (topological) Bardeen term to the boundary theory

$$
S_{B}=c_{B} \int d^{4} x \epsilon^{\mu \nu \lambda \rho} A_{\mu}^{A} A_{\nu}^{V} F_{\lambda \rho}^{V}
$$

Combining the corresponding Bardeen currents

$$
\begin{aligned}
j_{B}^{\mu} & =c_{B} \varepsilon^{\mu \nu \lambda \rho}\left(A_{\nu}^{V} F_{\lambda \rho}^{A}-2 A_{\nu}^{A} F_{\lambda \rho}^{V}\right), \\
j_{5, B}^{\mu} & =c_{B} \varepsilon^{\mu \nu \lambda \rho} A_{\nu}^{V} F_{\lambda \rho}^{V},
\end{aligned}
$$

with the vector and axial currents

$$
j^{\prime \mu} \equiv j^{\mu}+j_{B}^{\mu}, \quad j_{5}^{\prime \mu} \equiv j_{5}^{\mu}+j_{5, B}^{\mu},
$$

we obtain the anomaly equations

$\partial_{\mu} j^{\prime \mu}=-\left(\frac{C^{122}}{2}+c_{B}\right) F_{\alpha \beta}^{V} \tilde{F}^{A \alpha \beta}$

$\partial_{\mu} j_{5}^{\prime \mu}=-\frac{C^{111}}{4} F_{\alpha \beta}^{A} \tilde{F}^{A \alpha \beta}-\left(\frac{C^{122}}{4}-c_{B}\right) F_{\alpha \beta}^{V} \tilde{F}^{V \alpha \beta}$.

The electric current $j^{\prime \mu}$ is conserved if $c_{B}=-C^{122} / 2$. Setting $C^{111}=C^{122} \equiv C / 3$, the hydrodynamic Eqs. (3) become 


$$
\begin{aligned}
\partial_{\mu} T^{\mu \nu} & =F^{V \nu \lambda} j_{\lambda}^{\prime}+F^{A \nu \lambda} j_{5 \lambda}^{\prime}, \quad \partial_{\mu} j^{\prime \mu}=0, \\
\partial_{\mu} j_{5}^{\prime \mu} & =C E \cdot B+(C / 3) E_{5} \cdot B_{5} .
\end{aligned}
$$

Using the derivative expansion

$$
j^{\prime \mu}=\rho u^{\mu}+\kappa_{\omega} \omega^{\mu}+\kappa_{B} B^{\mu}+\kappa_{5, B} B_{5}^{\mu},
$$

where $\kappa_{\omega} \equiv \xi_{\omega}^{2}, \kappa_{B} \equiv \xi_{B}^{22}$ and $\kappa_{5, B} \equiv \xi_{B}^{21}$, we obtain from (28) and (29) the conductivities $\left(\mu_{5} \equiv \mu^{1}, \mu \equiv \mu^{2}\right)$

$$
\begin{aligned}
\kappa_{\omega} & =2 C \mu_{5}\left(\mu-\frac{\rho}{\epsilon+P_{T}}\left[\mu^{2}+\frac{\mu_{5}^{2}}{3}\right]\right), \\
\kappa_{B} & =C \mu_{5}\left(1-\frac{\mu \rho}{\epsilon+P_{T}}\right), \\
\kappa_{5, B} & =C \mu\left(1-\frac{1}{2} \frac{\mu \rho}{\epsilon+P_{T}}\left[1+\frac{\mu_{5}^{2}}{3 \mu^{2}}\right]\right) .
\end{aligned}
$$

There are analogous transport coefficients in the axial current $j_{5}^{\mu}$ [20]. The axial fields $E_{5 \mu}$ and $B_{5 \mu}$ are not needed and can now be switched off. The first term in $\kappa_{B}$ and $\kappa_{\omega}, \kappa_{B}=C \mu_{5}$ and $\kappa_{\omega}=2 C \mu \mu_{5}$, is the leading term in the chiral magnetic (CME) [4,5] and chiral vortical effect [46], respectively. ${ }^{2}$ They are in agreement with those found in the isotropic case $[16,17,20]$. The second term proportional to $\rho /\left(\epsilon+P_{T}\right)$ actually depends on the dynamics of the fluid ${ }^{3}$ and therefore on $\varepsilon_{p}$.

The dependence of $\kappa_{B}$ on $\varepsilon_{p}$ can be made more visible by introducing an average pressure $\bar{P}=\left(2 P_{T}+P_{L}\right) / 3$ such that $\epsilon=3 \bar{P}$. Assuming $\varepsilon_{p}$ to be small (see Fig. 1), we expand the CME coefficient $\kappa_{B}$ to linear order in $\varepsilon_{p}$,

$$
\kappa_{B} \approx C \mu_{5}\left(1-\frac{\mu \rho}{\epsilon+\bar{P}}\left[1-\frac{\varepsilon_{p}}{6}\right]\right) .
$$

At freeze-out the elliptic flow coefficient $v_{2} \approx \varepsilon_{p} / 2$ [25]. For small momentum anisotropies, the CME thus increases linearly in $v_{2}$.

\section{FLUID-GRAVITY MODEL}

In this section we construct the gravity dual of a static anisotropic plasma with diagonal stress-energy momentum $T_{\mu \nu}=\operatorname{diag}\left(\epsilon, P_{T}, P_{T}, P_{L}\right)$ and charge densities $\rho^{a}$.

We start from a five-dimensional $U(1)^{n}$ EinsteinMaxwell theory in an asymptotic AdS space. The action is

$$
\begin{aligned}
S= & \frac{1}{16 \pi G_{5}} \int d^{5} x \sqrt{-g}\left[R-2 \Lambda-F_{M N}^{a} F^{a M N}\right. \\
& \left.+\frac{S_{a b c}}{6 \sqrt{-g}} \varepsilon^{P K L M N} A_{P}^{a} F_{K L}^{b} F_{M N}^{c}\right],
\end{aligned}
$$

\footnotetext{
${ }^{2} \kappa_{5, B}$ represents another effect, which we added for completeness, but it seems not to be realized in heavy-ion collisions.

${ }^{3}$ In [15] this term was considered as a one-loop correction in an effective theory and $(\epsilon+P) / \rho$ was interpreted as the corresponding infrared cutoff in the energy/momentum integration.
}

where $\Lambda=-6$ is the cosmological constant. As usual, the $U(1)$ field strengths are defined by

$$
F_{M N}^{a}=\partial_{M} A_{N}^{a}-\partial_{N} A_{M}^{a},
$$

where $M, N, \ldots=0, \ldots, 4$ and $a=1, \ldots, n$. The ChernSimons term $A \wedge F \wedge F$ encodes the information of the triangle anomalies in the field theory [14]. In fact, the Chern-Simons coefficients $S_{a b c}$ are related to the anomaly coefficients $C_{a b c}$ by

$$
C_{a b c}=S_{a b c} /\left(4 \pi G_{5}\right) \text {. }
$$

The corresponding equations of motion are given by the combined system of Einstein-Maxwell and Maxwell equations,

$$
\begin{gathered}
G_{M N}-6 g_{M N}=T_{M N}, \\
\nabla_{M} F^{a M P}=-\frac{S_{a b c}}{8 \sqrt{-g}} \varepsilon^{P M N K L} F_{M N}^{b} F_{K L}^{c},
\end{gathered}
$$

where the energy-momentum tensor $T_{M N}$ is

$$
T_{M N}=-2\left(F_{M R}^{a} F_{N}^{a R}+\frac{1}{4} g_{M N} F_{S R}^{a} F^{a S R}\right) .
$$

\section{A. AdS black hole with multiple $U(1)$ charges}

A gravity dual to an isotropic fluid $(\epsilon=3 P)$ with multiple chemical potentials $\mu_{a}(a=1, \ldots, n)$ at finite temperature $T$ is given by an AdS black hole solution with mass $m$ and multiple $U(1)$ charges $q^{a}$. In EddingtonFinkelstein coordinates, the metric and $U(1)$ gauge fields of this solution are

$d s^{2}=-f(r) d t^{2}+2 d r d t+r^{2} d \vec{x}^{2}, \quad A^{a}=-A_{0}^{a}(r) d t$,

where

$$
f(r)=r^{2}-\frac{m}{r^{2}}+\sum_{a} \frac{\left(q^{a}\right)^{2}}{r^{4}}, \quad A_{0}^{a}(r)=\mu_{\infty}^{a}+\frac{\sqrt{3} q^{a}}{2 r^{2}} .
$$

The constants $\mu_{\infty}^{a}$ can be fixed such that the gauge fields vanish at the horizon. In case of a single charge $(n=1)$, the background reduces to an ordinary Reissner-Nordstrøm black hole solution in $\mathrm{AdS}_{5}$ [47].

The temperature $T$ and chemical potentials $\mu^{a}$ of the fluid are defined by

$$
\begin{gathered}
T=\frac{\kappa}{2 \pi}=\frac{f^{\prime}\left(r_{+}\right)}{4 \pi}=\frac{2 r_{+}^{6}-\sum_{a}\left(q_{a}\right)^{2}}{2 \pi r_{+}^{5}}, \\
\mu^{a}=A_{0}^{a}\left(r_{+}\right)-A_{0}^{a}\left(r_{\infty}\right),
\end{gathered}
$$

where $r_{+}$is the outer horizon defined by the maximal solution of $f(r)=0$, and $r_{\infty}$ indicates the location of the boundary. The temperature of the fluid is the Hawking temperature of the black hole and is computed from 
the surface gravity $\kappa=\left.\sqrt{\partial_{M}|\chi| \partial^{M}|\chi|}\right|_{r_{+}}$, where $|\chi|=$ $\left(-\chi^{M} \chi_{M}\right)^{(1 / 2)}$ is the norm of the timelike Killing vector $\chi^{M}=\delta_{0}^{M}[$ here $|\chi|=\sqrt{f(r)}]$.

\section{B. Anisotropic AdS geometry with multiple $U(1)$ charges}

We now construct a solution for an anisotropic fluid $\left(\epsilon=2 P_{T}+P_{L}\right)$. An ansatz for an anisotropic AdS black hole solution is given by

$$
\begin{aligned}
d s^{2}= & -f(r) d t^{2}+2 d r d t+r^{2}\left(w_{T}(r) d x^{2}+w_{T}(r) d y^{2}\right. \\
& \left.+w_{L}(r) d z^{2}\right), \\
A^{a}= & -A_{0}^{a}(r) d t .
\end{aligned}
$$

The anisotropies are realized via $w_{T}(r)$ and $w_{L}(r)$, which are functions of the momentum anisotropy $\varepsilon_{p}$ as defined in (2),

$$
\varepsilon_{p}=\frac{\left\langle P_{T}-P_{L}\right\rangle}{\left\langle P_{T}+P_{L}\right\rangle} .
$$

In the isotropic case $\left(\varepsilon_{p}=0\right)$, these functions are required to be one, $w_{T}(r)=w_{L}(r)=1$, and the background reduces to the AdS black hole geometry (47).

An analytical solution of the type (51) is difficult to find, and we resort to numerics in the next subsection. For this, we need to know the solution close to the boundary. An asymptotic solution $(r \rightarrow \infty)$ is given by the four functions

$$
\begin{aligned}
A_{0}^{a}(r) & =\mu_{\infty}^{a}+\frac{\sqrt{3} q^{a}}{2 r^{2}}+\mathcal{O}\left(r^{-8}\right), \\
f(r) / r^{2} & =1-\frac{m}{r^{4}}+\sum_{a} \frac{\left(q^{a}\right)^{2}}{r^{6}}+\mathcal{O}\left(r^{-8}\right), \\
w_{T}(r) & =1+\frac{w_{T}^{(4)}}{r^{4}}+\mathcal{O}\left(r^{-8}\right), \\
w_{L}(r) & =1+\frac{w_{L}^{(4)}}{r^{4}}+\mathcal{O}\left(r^{-8}\right),
\end{aligned}
$$

where $w_{L}^{(4)}=-2 w_{T}^{(4)}=-m \zeta / 2, \mu_{\infty}^{a}=$ const, and $\zeta$ is related to the momentum anisotropy $\varepsilon_{p}$ by

$$
\zeta=\frac{2 \varepsilon_{p}}{\varepsilon_{p}+3}
$$

The functions $w_{T}(r)$ and $w_{L}(r)$ have been introduced in view of the structure of the anisotropic fluid stress-energy tensor. More precisely, in (53) we fixed the $r^{-4}$ coefficients $w_{T}^{(4)}$ and $w_{L}^{(4)}$ such that the fluid stress-energy tensor is of the diagonal form (7), $T^{\mu \nu}=\operatorname{diag}\left(\epsilon, P_{T}, P_{T}, P_{L}\right)$ with $\epsilon=$ $2 P_{T}+P_{L}$. Computing the stress-energy tensor in the standard way from the asymptotic solution (53) via the extrinsic curvature, see e.g. [48], we find the transverse and longitudinal pressures

$$
P_{T}=\frac{m-4 w_{T}^{(4)}-4 w_{L}^{(4)}}{16 \pi G_{5}}=\frac{m(1+\zeta)}{16 \pi G_{5}},
$$

$$
P_{L}=\frac{m-8 w_{T}^{(4)}}{16 \pi G_{5}}=\frac{m(1-2 \zeta)}{16 \pi G_{5}} .
$$

Note that if (54) holds true, the pressures $P_{T}$ and $P_{L}$ satisfy (52). Likewise, the charge densities are

$$
\rho^{a}=\frac{\sqrt{3} q^{a}}{16 \pi G_{5}} .
$$

From these relations, we find the useful identity

$$
\frac{\rho^{a}}{\epsilon+P_{T}}=\frac{\sqrt{3} q^{a}}{4 m\left(1+\frac{1}{4} \zeta\right)},
$$

which we will need later.

\section{Numerical solution}

We now use shooting techniques to solve the system of ordinary differential equations (ODE) which follows from the equations of motion (44) and (45) upon substituting the ansatz (51). The idea is to vary the metric and gauge fields at some minimal value $r_{+}$in the radial direction, integrate outwards and find solutions with the correct asymptotic behavior (53). A similar method was previously applied in [40].

We first need to study the asymptotic solution near $r_{+}$ and near the boundary at $r_{\infty} \gg r_{+}$(we choose $r_{\infty}=50$ in our numerics). We define $r_{+}$by the maximal solution of

$$
f\left(r_{+}\right)=0
$$

and use scale invariance to set $r_{+}=1$. We then expand the functions in the metric and gauge fields near $r_{+}$in powers of the parameter $\varepsilon=\frac{r}{r_{+}}-1 \ll 1$ and substitute them into the equations of motion. In this way, we find that the only independent variables are $\left\{f^{\prime}\left(r_{+}\right), w_{T}\left(r_{+}\right)\right.$, $\left.w_{L}\left(r_{+}\right), w_{L}^{\prime}\left(r_{+}\right)\right\}$since the gauge field parameters $A_{0}^{a}\left(r_{+}\right)$ can be set to zero using gauge invariance, $A_{0}^{a}\left(r_{+}\right)=0$. The other parameters at $r_{+}$can be expressed in terms of these four parameters, e.g. $w_{T}^{\prime}\left(r_{+}\right)=w_{T}\left(r_{+}\right) w_{L}^{\prime}\left(r_{+}\right) / w_{L}\left(r_{+}\right)$.

The near-boundary solution is given by (51) with (53) and is parameterized by the values $\left(\zeta, m, q^{a}, \mu_{\infty}^{a}\right)$. The final set of data is summarized in the following table:

\begin{tabular}{ll}
\hline \hline$r=r_{+}=1$ & $r=r_{\infty} \gg r_{+}$ \\
\hline$A_{0}^{a}\left(r_{+}\right)=0$ & $\mu_{\infty}^{a}$ \\
$f\left(r_{+}\right)=0$ & $f\left(r_{\infty}\right)$ \\
$f^{\prime}\left(r_{+}\right)=$fixed & $A_{0}^{a \prime}\left(r_{\infty}\right)$ \\
$w_{L}\left(r_{+}\right)=$var & $w_{L}\left(r_{\infty}\right)$ \\
$w_{T}\left(r_{+}\right)=$var & $w_{T}\left(r_{\infty}\right)$ \\
$w_{L}^{\prime}\left(r_{+}\right)=$var & \\
\hline \hline
\end{tabular}

Parameters not listed are related to those in the table by the equations of motion.

To integrate the equations we proceed as follows. We fix $\zeta$ and vary three parameters at $r_{+}$, namely $w_{T}\left(r_{+}\right), w_{L}\left(r_{+}\right)$ and $w_{L}^{\prime}\left(r_{+}\right)$, by choosing a grid with suitable number of 
sites (in our case $20^{3}-40^{3}$ ). The value $f^{\prime}\left(r_{+}\right)$can be thought of as the temperature of the system and will simply be fixed to some value. It turns out that the form of the functions $w_{L, T}(r)$ does not depend on this parameter. For each site in the grid we numerically solve the system of ODEs and determine the pair $\left(m, q^{a}\right)$ from the known asymptotics of $A_{0}^{a \prime}\left(r=r_{\infty}\right)$ and $f\left(r=r_{\infty}\right)$. This ensures that the analytical and numerical values for these quantities coincide.

We then calculate the combined residual

$$
\begin{aligned}
& \left.\operatorname{res}_{\infty}\left[w_{T}\left(r_{+}\right), w_{L}\left(r_{+}\right), w_{L}^{\prime}\left(r_{+}\right)\right)\right] \\
& \quad=\left(w_{L}^{\#}\left(r_{\infty}\right)-w_{L}^{*}\left(r_{\infty}\right)\right)^{2}+\left(w_{T}^{\#}\left(r_{\infty}\right)-w_{T}^{*}\left(r_{\infty}\right)\right)^{2},
\end{aligned}
$$

where $w_{L, T}^{\#}\left(r_{\infty}\right)$ are the numerical values, and $w_{L, T}^{*}\left(r_{\infty}\right)$ are the analytical values given by (53). We interpolate the residual by a piecewise linear function and find its global minimum by the simulated annealing method [49]. The result of the minimization is shown in Fig. 3, which depicts numerical plots of $f(r), A_{0}(r), w_{T}(r)$ and $w_{L}(r)$ for $n=1$.

We conclude this section with a comment on $r_{+}$. In the isotropic case, $r_{+}$is simply the size of the horizon of the AdS black hole geometry. For nonvanishing anisotropies and vanishing $U(1)$ charges, a naked singularity was found at $r_{+}$[38], implying that the static background does not exist indefinitely. The singularity is mild in the sense that there is a notion of ingoing boundary conditions and possible instabilities are absent at the linear level in the anisotropy parameter [38]. This behavior may persist even for nonvanishing $U(1)$ charges, even though it was
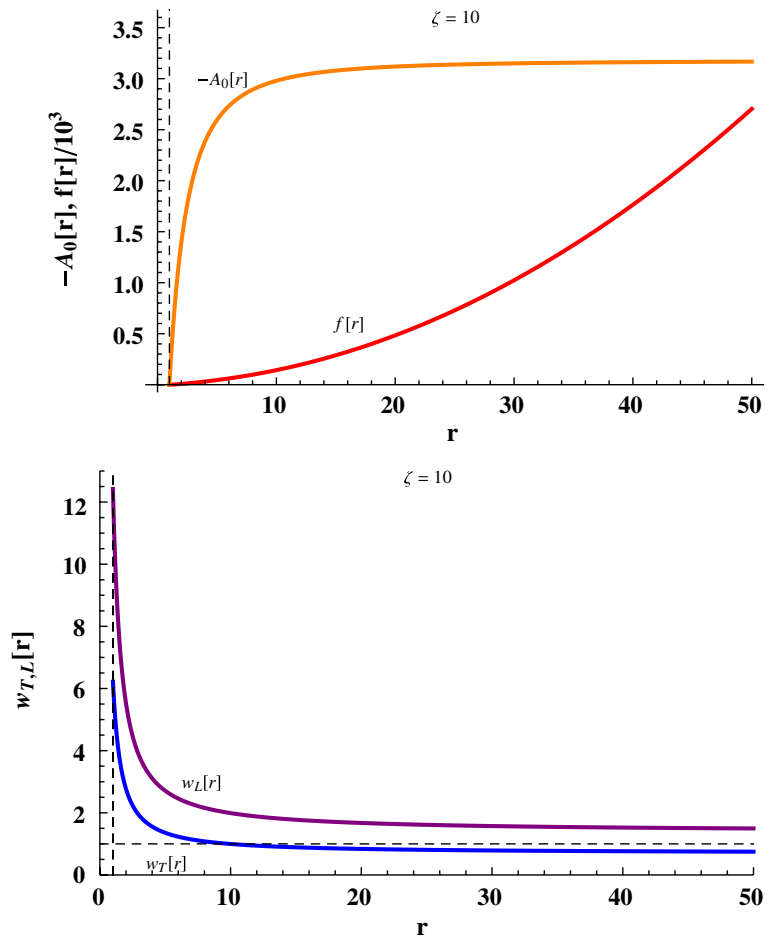

FIG. 3 (color online). Numerical plots of $f(r), A_{0}(r), w_{T}(r)$ and $w_{L}(r)$ for $\zeta=10\left(r_{+}=1\right)$. We get $w_{L}\left(r_{+}\right)=12.42$.

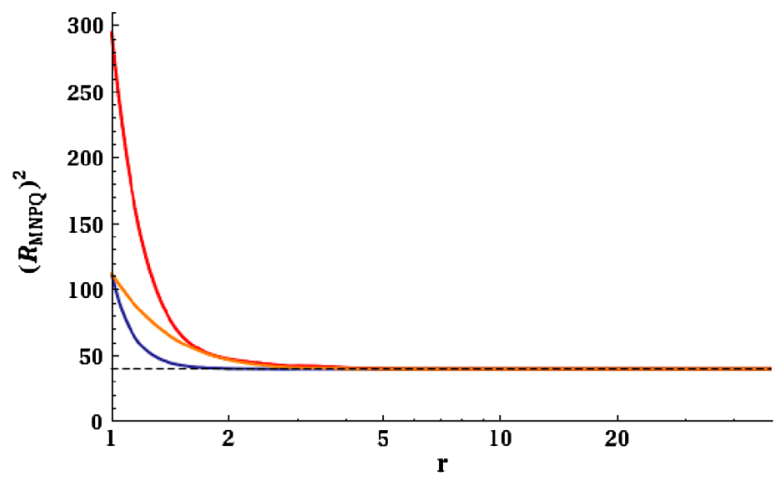

FIG. 4 (color online). Numerical plots of $\left(R_{M N P Q}\right)^{2}$ for $\zeta=10, q \neq 0$ (red), $\zeta=10, q=0$ (orange), and $\zeta=0$, $q=0$ (blue).

difficult to see the singularity in our numerics, cf. Figure 4. Despite this subtlety, we show in the next section that, at least for small anisotropies where the bulk geometry approximates a black hole solution, the singular geometry may be used to compute some transport coefficients of the fluid.

\section{HOLOGRAPHIC VORTICAL AND MAGNETIC CONDUCTIVITIES}

We will now compute the chiral vortical and magnetic conductivities $\xi_{\omega}^{a}$ and $\xi_{B}^{a b}$ from first-order corrections to the numerical AdS geometry (51) using the fluid-gravity correspondence [41].

\section{A. First-order corrected background}

In order to become a dual to a multiply charged fluid, the AdS geometry (51) must be boosted along the fourvelocity of the fluid $u_{\mu}(\mu=0, \ldots, 3)$. The boosted version of (51) is

$$
\begin{aligned}
d s^{2}= & \left(r^{2} w_{T}(r) P_{\mu \nu}-f(r) u_{\mu} u_{\nu}\right) d x^{\mu} d x^{\nu}-2 u_{\mu} d x^{\mu} d r \\
& -r^{2}\left(w_{T}(r)-w_{L}(r)\right) v_{\mu} v_{\nu} d x^{\mu} d x^{\nu}, A^{a} \\
= & \left(A_{0}^{a}(r) u_{\mu}+\mathcal{A}_{\mu}^{a}\right) d x^{\mu},
\end{aligned}
$$

where $P^{\mu \nu}=g^{\mu \nu}+u^{\mu} u^{\nu}$, and $f(r), A_{0}^{a}(r), w_{T}(r)$ and $w_{L}(r)$ are numerically known functions. As in hydrodynamics, the four-vector $v^{\mu}$ determines the direction of the longitudinal axis, cf. Sec. II. Following [14,20], we have formally introduced constant background gauge fields $\mathcal{A}_{\mu}^{a}$ to model external electromagnetic fields, such as the magnetic fields $B^{a \mu}$ needed for the chiral magnetic effect.

The transport coefficients $\xi_{\omega}^{a}$ and $\xi_{B}^{a b}$ can now be computed using standard fluid-gravity techniques [41]. We closely follow $[14,20,50]$, in which these transport coefficients were determined for an isotropic fluid with one and three charges $(n=1,3)$. We work in the static frame $u_{\mu}=(-1,0,0,0), v_{\mu}=(0,0,0,1)$, and consider vanishing background fields $\mathcal{A}_{\mu}^{a}$ (at $x^{\mu}=0$ ). The transport 
coefficients $\xi_{\omega}^{a}$ and $\xi_{B}^{a b}$ measure the response of the system to rotation and the perturbation by an external magnetic field. We therefore slowly vary the velocity $u_{\mu}$ and the background fields $\mathcal{A}_{\mu}^{a}$ up to first order as

$$
u_{\mu}=\left(-1, x^{\nu} \partial_{\nu} u_{i}\right), \quad \mathcal{A}_{\mu}^{a}=\left(0, x^{\nu} \partial_{\nu} \mathcal{A}_{i}^{a}\right) .
$$

We may also vary $m$ and $q$ in this way, but it turns out that varying these parameters has no influence on the transport coefficients $\xi_{\omega}^{a}$ and $\xi_{B}^{a b}$.

Because of the dependence on $x^{\mu}$, the background (61) is no longer an exact solution of the equations of motion. Instead with varying parameters the solution (61) receives higher-order corrections, which are in this case of first order in the derivatives.

An ansatz for the first-order corrected metric and gauge fields is given by

$$
\begin{aligned}
d s^{2}= & \left(-f(r)+\tilde{g}_{t t}\right) d t^{2}+2\left(1+\tilde{g}_{t r}\right) d t d r+r^{2}\left(w_{T}(r) d x^{2}\right. \\
& \left.+w_{T}(r) d y^{2}+w_{L}(r) d z^{2}\right)+\tilde{g}_{i j} d x^{i} d x^{j} \\
& -2 x^{\nu} \partial_{\nu} u_{i} d r d x^{i}+2\left(\left(f(r)-r^{2}\right) x^{\nu} \partial_{\nu} u_{i}+\tilde{g}_{t i}\right) d t d x^{i} \\
A^{a}= & \left(-A_{0}^{a}(r)+\tilde{A}_{t}^{a}\right) d t+\left(A_{0}^{a}(r) x^{\nu} \partial_{\nu} u_{i}\right. \\
& \left.+x^{\nu} \partial_{\nu} \mathcal{A}_{i}^{a}+\tilde{A}_{i}^{a}\right) d x^{i},
\end{aligned}
$$

where the first-order corrections are denoted by

$$
\tilde{g}_{M N}=\tilde{g}_{M N}(r), \quad \tilde{A}_{M}^{a}=\tilde{A}_{M}^{a}(r) .
$$

As in [50], we work in the gauge

$$
\tilde{g}_{r r}=0, \quad \tilde{g}_{r \mu} \sim u_{\mu}, \quad \tilde{A}_{r}^{a}=0, \quad \sum_{i=1}^{3} \tilde{g}_{i i}=0 .
$$

The first-order corrections can be obtained by substituting the ansatz (63) into the equations of motion (44) and (45). The computation is straightforward but lengthy and has been shifted to Appendix C [we set $\mu_{\infty}^{a}=A_{0}^{a}\left(r_{\infty}\right)=0$ there, see Sec. II C for a discussion]. As a result, we find the following corrections:

$$
\begin{aligned}
\tilde{g}_{t r}= & \tilde{g}_{t t}=\tilde{A}_{t}^{a}=0, \\
\tilde{g}_{t i}(r)= & f(r) \int_{\infty}^{r} d r^{\prime} \frac{1}{w_{L}\left(r^{\prime}\right)^{1 / 2} r^{\prime}\left(f\left(r^{\prime}\right)\right)^{2}} \\
& \times\left(\int_{r_{+}}^{r^{\prime}} d r^{\prime \prime} I\left(r^{\prime \prime}\right)-w_{L}\left(r_{+}\right)^{1 / 2} r_{+} f^{\prime}\left(r_{+}\right) C_{i}\right), \\
\tilde{A}_{i}^{a}(r)= & \int_{\infty}^{r} d r^{\prime} \frac{1}{r^{\prime} f\left(r^{\prime}\right) w_{L}\left(r^{\prime}\right)^{1 / 2}}\left[Q_{i}^{a}\left(r^{\prime}\right)-Q_{i}^{a}\left(r_{+}\right)\right. \\
& \left.-C_{i} r_{+} A_{0}^{a \prime}\left(r_{+}\right) w_{L}\left(r_{+}\right)^{1 / 2}+r^{\prime} \tilde{g}_{t i}\left(r^{\prime}\right) A_{0}^{a \prime}\left(r^{\prime}\right)\right],
\end{aligned}
$$

with

$$
\begin{aligned}
I(r)= & \sum_{a=1}^{n} 4 A_{0}^{a \prime}(r)\left(Q_{i}^{a}(r)-Q_{i}^{a}\left(r_{+}\right)\right. \\
& \left.-C_{i} r_{+} w_{L}\left(r_{+}\right)^{1 / 2} A_{0}^{a \prime}\left(r_{+}\right)\right), \\
Q_{a}^{i} \equiv & \frac{1}{2} S_{a b c} A_{0}^{b} A_{0}^{c} \epsilon^{i j k}\left(\partial_{j} u_{k}\right)+S_{a b c} A_{0}^{b} \epsilon^{i j k}\left(\partial_{j} \mathcal{A}_{k}^{c}\right), \\
C^{i}= & \frac{4 c\left(r_{+}\right)}{w_{L}\left(r_{+}\right)^{1 / 2}}\left(\frac{1}{3} S_{a b c} A_{0}^{a}\left(r_{+}\right) A_{0}^{b}\left(r_{+}\right) A_{0}^{c}\left(r_{+}\right) \epsilon^{i j k}\left(\partial_{j} u_{k}\right)\right. \\
& \left.+\frac{1}{2} S_{a b c} A_{0}^{a}\left(r_{+}\right) A_{0}^{b}\left(r_{+}\right) \epsilon^{i j k}\left(\partial_{j} \mathcal{A}_{k}^{c}\right)\right), \\
c\left(r_{+}\right)= & \frac{1}{r_{+}\left(f^{\prime}\left(r_{+}\right)-4 \sum_{a} A_{0}^{a}\left(r_{+}\right) A_{0}^{a \prime}\left(r_{+}\right)\right)},
\end{aligned}
$$

and $r_{+}$as defined around (59) $\left[\tilde{g}_{i j}\right.$ can be obtained by solving (C2) in Appendix $\mathrm{C}$ but will not be needed here].

\section{B. Holographic conductivities}

On the boundary of the asymptotic AdS space (63), the metric and gauge fields couple to the fluid stress-energy tensor and $U(1)$ currents, respectively. Holographic renormalization [51] provides relations between these currents and the near-boundary behavior of their dual bulk fields. For the magnetic and vortical effects, we need the $U(1)$ currents $j^{a \mu}$, which are related to the bulk gauge fields $A^{a \mu}$ by $[51,52]$

$$
j^{a \mu}=\lim _{r \rightarrow \infty} \frac{r^{2}}{8 \pi G_{5}} \eta^{\mu \nu} A_{\nu}^{a}(r) .
$$

Expanding the solution in $\frac{1}{r}$ and substituting only the corrections $\tilde{A}_{\mu}^{a}$, we get the currents

$$
\begin{aligned}
\tilde{j}^{a \mu} & =\lim _{r \rightarrow \infty} \frac{r^{2}}{8 \pi G_{5}} \eta^{\mu \nu} \tilde{A}_{\nu}^{a}(r) \\
& =\frac{1}{16 \pi G_{5}} \eta^{\mu \nu}\left(Q_{\nu}^{a}\left(r_{+}\right)+r_{+} A_{0}^{a l}\left(r_{+}\right) C_{\nu}\right) .
\end{aligned}
$$

Note that, in the isotropic case $\left(w_{L}=1, P_{T}=P_{L}=P\right)$, the prefactor of the second term of (68) is simply

$$
r_{+} A_{0}^{a \prime}\left(r_{+}\right) c\left(r_{+}\right)=\frac{\sqrt{3}}{4 m} q^{a},
$$

as can be seen by substituting the Reissner-Nordstrøm solution (48) into the left-hand side of this equation. In the anisotropic case, we need to show that

$$
r_{+} A_{0}^{a \prime}\left(r_{+}\right) c\left(r_{+}\right) \cdot w_{L}\left(r_{+}\right)^{-1 / 2}=\frac{\sqrt{3} q^{a}}{4 m} \cdot \frac{1}{1+\frac{1}{4} \zeta},
$$

which, by (58), is equivalent to $\rho^{a} /\left(\epsilon+P_{T}\right)$. This equation holds, in particular, if the first and second factors on both sides agree individually. The first factors correspond to (69), which is expected to hold, at least approximately for small anisotropies $\zeta$. The second factors are identical 


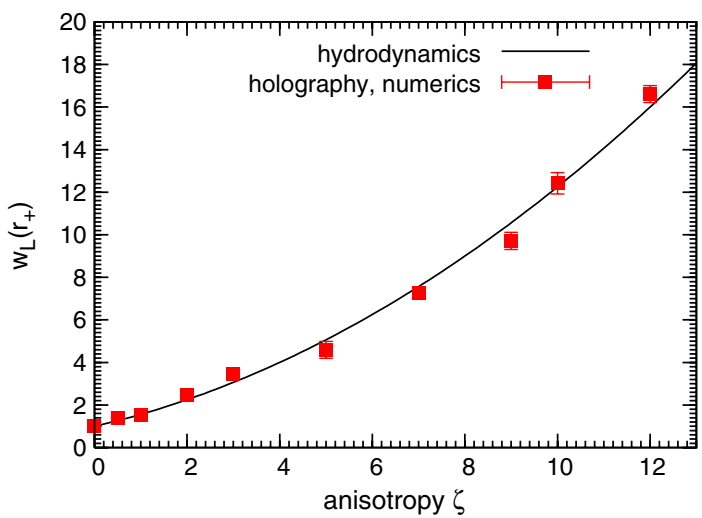

FIG. 5 (color online). Values of $w_{L}\left(r_{+}\right)$as a function of the anisotropy $\zeta$. The numerically determined values for $w_{L}\left(r_{+}\right)$lie on the solid curve, which represents the function $\left(1+\frac{1}{4} \zeta\right)^{2}$.

if $w_{L}\left(r_{+}, \zeta\right)=\left(1+\frac{1}{4} \zeta\right)^{2}$. We find numerically (for $n=1$ ) that $w_{L}\left(r_{+}\right)$indeed satisfies this equation, see Fig. 5. Thus (70) holds numerically, at least in the limit of small $\zeta$.

Comparing (68) with the general expansion

$$
\begin{aligned}
\tilde{j}^{a \mu} & =\xi_{\omega}^{a} \omega^{\mu}+\xi_{B}^{a b} B^{b \mu} \\
& =\xi_{\omega}^{a} \frac{1}{2} \epsilon^{\nu \rho \sigma \mu} u_{\nu} \partial_{\rho} u_{\sigma}+\xi_{B}^{a b} \epsilon^{\nu \rho \sigma \mu} u_{\nu} \partial_{\rho} \mathcal{A}_{\sigma}^{b},
\end{aligned}
$$

we finally obtain the coefficients

$$
\begin{gathered}
\xi_{\omega}^{a}=\frac{4}{16 \pi G_{5}}\left(S^{a b c} \mu^{b} \mu^{c}-\frac{2}{3} \frac{\rho^{a}}{\epsilon+P_{T}} S^{b c d} \mu^{b} \mu^{c} \mu^{d}\right), \\
\xi_{B}^{a b}=\frac{4}{16 \pi G_{5}}\left(S^{a b c} \mu^{c}-\frac{1}{2} \frac{\rho^{a}}{\epsilon+P_{T}} S^{b c d} \mu^{c} \mu^{d}\right),
\end{gathered}
$$

with $\mu^{a} \equiv A_{0}^{a}\left(r_{+}\right)$[since $A_{0}^{a}(\infty)=0$ ]. Using the relation (43), we find that the holographically computed transport coefficients (72) and (73) coincide exactly with those found in hydrodynamics, (28) and (29).

\section{Subtleties in holographic descriptions of the CME}

The conservation of the electromagnetic current requires the introduction of the Bardeen counterterm into the action. In AdS/QCD models of the CME, this typically leads to a vanishing result for the electromagnetic current $[29,31]$. The problem is related to the difficulty of introducing a chemical potential conjugated to a nonconserved chiral charge $[29,30]$. It is possible to modify the action to obtain a conserved chiral charge [29]. This charge is however only gauge-invariant when integrated over all space in homogeneous configurations.

In AdS black hole models of the CME, one usually introduces a chiral chemical potential dual to a gaugeinvariant current, despite it being anomalous [20,30]. The prize to pay is the appearance of a singular bulk gauge field at the horizon, a phenomenon which seems to be generic in AdS black hole models of the CME.

Careful holographic renormalization shows that, in the presence of Chern-Simons terms, there is an additional term on the right-hand side of (67) [52]. This term is of the form

$$
\hat{j}_{a}^{\mu}=-\frac{S_{a b c}}{8 \pi G_{5}} \epsilon^{\mu \nu \rho \sigma} A_{b \nu}^{(0)}(x) \partial_{\rho} A_{c \sigma}^{(0)}(x),
$$

where $A_{a \mu}^{(0)}(x)$ are the 0 th-order coefficients in a $\frac{1}{r}$ expansion of the bulk gauge fields $A_{a \mu}(r, x)$. In (62) we expanded the background gauge fields $\mathcal{A}_{\mu}^{a}$ around zero and set $A_{\nu}^{a(0)}=\mu_{\infty}^{a} u_{\nu}=0$. This allowed us to ignore terms in (67) coming from (74) (at least to first order in the derivatives).

Problems arise if $\mu_{\infty}^{a} \neq 0$. To see this, let us restrict again to two charges $(n=2)$ as in Sec. II C and define axial and vector gauge fields by $A_{\mu}^{A}=A_{\mu}^{1}$ and $A_{\mu}^{V}=A_{\mu}^{2}$. Then $\hat{j}^{\mu}=\hat{j}_{2}^{\mu}$ gives rise to additional contributions of the type

$$
\hat{j}^{\mu} \supset \varepsilon^{\mu \nu \rho \sigma} A_{\nu}^{A(0)}(x) F_{\rho \sigma}^{V(0)}(x),
$$

which are forbidden by electromagnetic gauge invariance [29], unless $A_{\nu}^{A(0)}(x)=0$. However, in general $A_{\nu}^{A(0)}(x)=$ $\mu_{5}^{\infty} u_{\nu}($ at $x=0)$ with some constant $\mu_{5}^{\infty}$. We should thus set $\mu_{5}^{\infty}=0$ [Note that this does not imply $\mu_{5}=$ $\left.A_{0}^{A}\left(r_{\infty}\right)-A_{0}^{A}\left(r_{+}\right)=0\right]$. This corresponds to a nonvanishing gauge field at the horizon, as noticed also in $[20,30]$.

\section{CONCLUSIONS}

Our main result is (40), which gives the chiral magnetic conductivity $\kappa_{B}$ for an anisotropic plasma. It explicitly shows the dependence on the momentum anisotropy $\varepsilon_{p}$. We also computed the CME coefficient in the holographic dual model and found numerical agreement with the hydrodynamic result for small anisotropies.

\section{ACKNOWLEDGMENTS}

We thank Johanna Erdmenger, Patrick Kerner and Andreas Schäfer for discussions and helpful comments. I. K. is grateful to Berndt Müller for pointing out Ref. [13] and Quan Wang for answering questions related to the measurement of the charge separation as a function of $v_{2}$. I. G. would like to thank Shi $\mathrm{Pu}$ for email correspondence.

\section{APPENDIX A: COMPUTATION OF $\partial_{\mu} \omega^{\mu}$ TEXT AND $\partial_{\mu} B^{\mu}$ TEXT}

In the following we will use the identities

$$
\begin{gathered}
u^{\mu} u^{\lambda} \partial_{\mu} \omega_{\lambda}=-\frac{1}{2} \partial_{\mu} \omega^{\mu}, \\
u^{\mu} u^{\lambda} \partial_{\mu} B_{\lambda}=\partial_{\mu} B^{\mu}+2 \omega^{\rho} E_{\rho} .
\end{gathered}
$$


To find an explicit expression for $\partial_{\mu} \omega^{\mu}$, we compute the term $\omega_{\nu} \partial_{\mu} T^{\mu \nu}$ in two ways. First, using the hydrodynamic equations, we get

$$
\omega_{\nu} \partial_{\mu} T^{\mu \nu}=\omega_{\nu} F^{\nu \mu} j_{\mu}=\rho \omega_{\nu} F^{\nu \mu} u_{\mu}=\rho \omega_{\nu} E^{\nu} .
$$

Next, substituting the stress-energy tensor (4) in this expression, we find

$$
\begin{aligned}
\omega_{\nu} \partial_{\mu} T^{\mu \nu}= & \left(\epsilon+P_{T}\right) u^{\mu} \omega_{\nu} \partial_{\mu} u^{\nu}+\omega_{\nu} g^{\mu \nu} \partial_{\mu} P_{T} \\
& -\Delta \omega_{\nu} v^{\mu} \partial_{\mu} v^{\nu}-v^{\nu} \omega_{\nu} v^{\mu} \partial_{\mu} \Delta \\
& -\Delta v^{\nu} \omega_{\nu} \partial_{\mu} v^{\mu} \\
= & -\left(\epsilon+P_{T}\right) u^{\mu} u^{\nu} \partial_{\mu} \omega_{\nu}+\omega^{\mu} \partial_{\mu} P_{T} \\
& -\Delta \omega_{\nu} \partial^{\nu} \ln \tau-v^{\nu} \omega_{\nu} v^{\mu} \partial_{\mu} \Delta \\
& -\Delta v^{\nu} \omega_{\nu} \partial_{\mu} v^{\mu} .
\end{aligned}
$$

Using the identity (A1), we find

$$
\begin{aligned}
\partial_{\mu} \omega^{\mu}= & -\frac{2}{\epsilon+P_{T}} \omega^{\mu}\left(\partial_{\mu} P_{T}-\Delta \partial_{\mu} \ln \tau-\rho E_{\mu}\right. \\
& \left.-v_{\mu} v^{\nu} \partial_{\nu} \Delta-\Delta v_{\mu} \partial_{\nu} v^{\nu}\right) .
\end{aligned}
$$

Similar manipulations of the term $B_{\nu} \partial_{\mu} T^{\mu \nu}$ lead to

$$
\begin{aligned}
& B_{\nu} \partial_{\mu} T^{\mu \nu}=B_{\nu} F^{\nu \mu} j_{\mu}=\rho B_{\mu} E^{\mu} \\
B_{\nu} \partial_{\mu} T^{\mu \nu}= & -\left(\epsilon+P_{T}\right) u^{\mu} u^{\nu} \partial_{\mu} B_{\nu}+B^{\mu} \partial_{\mu} P_{T} \\
& -\Delta B_{\nu} v^{\mu} \partial_{\mu} v^{\nu}-B_{\nu} v^{\nu} v^{\mu} \partial_{\mu} \Delta \\
& -\Delta B_{\nu} v^{\nu} \partial_{\mu} v^{\mu} \\
= & -\left(\epsilon+P_{T}\right)\left(\partial_{\mu} B^{\mu}-2 \omega^{\mu} E_{\mu}\right)-\Delta B_{\mu} \partial^{\mu} \ln \tau \\
& -B_{\nu} v^{\nu} v^{\mu} \partial_{\mu} \Delta-\Delta B_{\nu} v^{\nu} \partial_{\mu} v^{\mu},
\end{aligned}
$$

where we used (A2). From (A6) and (A7) we obtain the following expression:

$$
\begin{aligned}
\partial_{\mu} B^{\mu}= & -2 \omega^{\mu} E_{\mu}-\frac{B^{\mu}}{\epsilon+P_{T}}\left(\partial_{\mu} P_{T}-\Delta \partial_{\mu} \ln \tau\right. \\
& \left.-\rho E_{\mu}-v_{\mu} v^{\nu} \partial_{\nu} \Delta-\Delta v_{\mu} \partial_{\nu} v^{\nu}\right) .
\end{aligned}
$$

The last two terms in (A5) and (A8) vanish provided the fluid satisfies

$$
\partial_{\mu} v^{\mu}=0, \quad v^{\mu} \partial_{\mu} \Delta=0 .
$$

Then (A5) and (A8) become identical to the expressions in (19).

\section{APPENDIX B: COMPUTATION OF THE TRANSPORT COEFFICIENTS $\xi_{\omega}$ TEXT AND $\xi_{B}$ TEXT}

In this appendix we compute the conductivities $\xi_{\omega}$ and $\xi_{B}$ by solving the system of Eqs. (22)-(25). Following [14], we change variables from $\ln \tau, \mu, T$ to $\ln \tau$, $\bar{\mu}=\mu / T$ and $P_{T}$. From (12) and (13), we derive the thermodynamic expressions

$$
\begin{gathered}
\left(\frac{\partial \bar{\mu}}{\partial T}\right)_{P_{T}, \ln \tau}=-\frac{\epsilon+P_{T}}{\rho T^{2}}, \\
\left(\frac{\partial P_{T}}{\partial T}\right)_{\bar{\mu}, \ln \tau}=\frac{\epsilon+P_{T}}{T}, \\
\left(\frac{\partial \ln \tau}{\partial T}\right)_{\bar{\mu}, P_{T}}=-\frac{1}{\Delta} \frac{\epsilon+P_{T}}{T} .
\end{gathered}
$$

Using

$$
\begin{gathered}
\partial_{\mu} D=\frac{\partial D}{\partial P_{T}} \partial_{\mu} P_{T}+\frac{\partial D}{\partial \bar{\mu}} \partial_{\mu} \bar{\mu}+\frac{\partial D}{\partial \ln \tau} \partial_{\mu} \ln \tau, \\
\partial_{\mu} D_{B}=\frac{\partial D_{B}}{\partial P_{T}} \partial_{\mu} P_{T}+\frac{\partial D_{B}}{\partial \bar{\mu}} \partial_{\mu} \bar{\mu}+\frac{\partial D_{B}}{\partial \ln \tau} \partial_{\mu} \ln \tau,
\end{gathered}
$$

the first two equations, (22) and (23), can be rewritten as

$$
\begin{array}{rlrl}
-\xi_{\omega}+\frac{\partial D}{\partial \bar{\mu}}=0, & & -\xi_{B}+\frac{\partial D_{B}}{\partial \bar{\mu}}=0, \\
\frac{\partial D}{\partial P_{T}}-\frac{2 D}{\epsilon+P_{T}}=0, & \frac{\partial D_{B}}{\partial P_{T}}-\frac{D_{B}}{\epsilon+P_{T}}=0, \\
\frac{\partial D}{\partial \ln \tau}+\frac{2 \Delta D}{\epsilon+P_{T}}=0, & \frac{\partial D_{B}}{\partial \ln \tau}+\frac{\Delta D_{B}}{\epsilon+P_{T}}=0 .
\end{array}
$$

Note that (B7) and (B8) are related by the thermodynamic identities (B2) and (B3). Using the ansatz

$$
D=T^{2} d(\bar{\mu}, \ln \tau), \quad D_{B}=T d_{B}(\bar{\mu}, \ln \tau),
$$

and (B1), we obtain two differential equations from (24) and (25),

$$
\begin{gathered}
0=\frac{2 \rho D}{\epsilon+P_{T}}-2 D_{B}+\frac{\xi_{\omega}}{T} \\
\left.=T\left(\partial_{\bar{\mu}} d(\bar{\mu}, \ln \tau)-2 d_{B}(\bar{\mu}, \ln \tau)\right)\right), \\
0=\frac{\rho D_{B}}{\epsilon+P_{T}}+\frac{\xi_{B}}{T}-C \bar{\mu}=\partial_{\bar{\mu}} d_{B}(\bar{\mu}, \ln \tau)-C \bar{\mu} .
\end{gathered}
$$

These equations can be integrated to give

$$
d_{B}(\bar{\mu}, \ln \tau)=\frac{1}{2} C \bar{\mu}^{2}+\beta(\ln \tau),
$$

$$
d(\bar{\mu}, \ln \tau)=\frac{1}{3} C \bar{\mu}^{3}+2 \bar{\mu} \beta(\ln \tau)+\gamma(\ln \tau),
$$

where $\beta(\ln \tau)$ and $\gamma(\ln \tau)$ are arbitrary functions of $\ln \tau$. Substituting this back into (24) and (25), we get the conductivities 


$$
\begin{aligned}
\xi_{\omega}= & C\left(\mu^{2}-\frac{2}{3} \frac{\rho \mu^{3}}{\epsilon+P_{T}}\right)+2 T^{2} \beta(\ln \tau) \\
& -\frac{2 \rho T^{3}}{\epsilon+P_{T}}(2 \bar{\mu} \beta(\ln \tau)+\gamma(\ln \tau)), \\
\xi_{B}= & C\left(\mu-\frac{1}{2} \frac{\rho \mu^{2}}{\epsilon+P_{T}}\right)-\frac{T^{2}}{\epsilon+P_{T}} \beta(\ln \tau) .
\end{aligned}
$$

The function $\gamma(\ln \tau)$ is forbidden by $C P T$ invariance [36].

\section{APPENDIX C: FIRST-ORDER CORRECTED BACKGROUND GEOMETRY}

In this appendix we compute the first-order corrections to the background (61) using the ansatz (63). The computation follows that for the three-charge STU model [26] presented in $[20,50]$.

We begin by substituting the ansatz (63) into the equations of motion (44) and (45). We denote the resulting Maxwell equations, Eqs. (45) by $M_{N}^{a}(a=1, \ldots, n)$ and the components of the Einstein equation, Eq. (44) by $E_{M N}$ $M, N=0, \ldots, 4 \quad\left[x^{M}=\left(t, x^{1}, x^{2}, x^{3}, r\right)\right]$. Then, from $g^{r t} E_{t i}+g^{r r} E_{r i}=0$, we find $\partial_{t} u_{i}=0$, and $E_{t t}, E_{r t}, E_{r r}$, $E_{t t}, M_{t}^{a}$, and $M_{r}^{a}$ are solved by

$$
\partial_{i} u_{i}=\tilde{g}_{t r}=\tilde{g}_{t t}=\tilde{A}_{t}^{a}=0 .
$$

The remaining equations are $E_{i j}, E_{t i}, M_{i}^{a}$.

From $E_{i j}$ we get

$$
-\partial_{r}\left(r^{3} f(r) \partial_{r}\left(\frac{\tilde{g}_{i j}(r)}{r^{2}}\right)\right)=3 r^{2}\left(\partial_{i} u_{j}+\partial_{j} u_{i}\right) .
$$

From $E_{t i}$ we get

$$
\begin{aligned}
& {\left[\frac{f^{\prime}(r)}{f(r)}\left(\frac{2}{r}+\frac{w_{T}^{\prime}(r)}{w_{T}(r)}\right)+\frac{4}{3 f(r)}\left(\sum_{a=1}^{n} A_{0}^{a \prime}(r)^{2}-6\right)\right] \tilde{g}_{t i}(r)} \\
& +\left(\frac{1}{r}+\frac{w_{L}^{\prime}(r)}{2 w_{L}(r)}\right) \tilde{g}_{t i}^{\prime}(r)+\tilde{g}_{t i}^{\prime \prime}(r)=4 \sum_{a=1}^{n} A_{0}^{a \prime}(r) \tilde{A}_{i}^{a \prime}(r),
\end{aligned}
$$

where a prime denotes the partial derivative $\partial_{r}$ with respect to $r$.

From $M_{i}^{a}$ we get

$$
\begin{aligned}
\partial_{r} & {\left[w_{L}(r)^{1 / 2} r\left(f(r) \tilde{A}_{i}^{a \prime}-\tilde{g}_{t i}(r) A_{0}^{a \prime}\right)\right] } \\
& =\partial_{r}\left(\frac{1}{2} S_{a b c} A_{0}^{b} A_{0}^{c} \epsilon^{i j k}\left(\partial_{j} u_{k}\right)+S_{a b c} A_{0}^{b} \epsilon^{i j k}\left(\partial_{j} \mathcal{A}_{k}^{c}\right)\right) \\
& \equiv \partial_{r} Q_{i}^{a}(r) .
\end{aligned}
$$

Equation (C2) depends only on $\tilde{g}_{i j}(r)$ and can easily be solved. The integration of (C4) leads to

$$
w_{L}(r)^{1 / 2}\left(r f(r) \tilde{A}_{i}^{a \prime}(r)-r \tilde{g}_{t i}(r) A_{0}^{a \prime}(r)\right)=Q_{i}^{a}(r)+C_{i}^{a} .
$$

Here $C_{i}^{a}$ are some integration constants, which can be fixed as

$$
C_{i}^{a}=-Q_{i}^{a}\left(r_{+}\right)-C_{i} w_{L}\left(r_{+}\right)^{1 / 2} r_{+} A_{0}^{a l}\left(r_{+}\right),
$$

with $r_{+}$as in (59) and $C_{i}=\tilde{g}_{t i}\left(r_{+}\right)$. This can be solved for $\tilde{A}_{i}^{a}(r)$,

$$
\begin{aligned}
\tilde{A}_{i}^{a}(r)= & \int_{\infty}^{r} d r^{\prime} \frac{1}{r^{\prime} f\left(r^{\prime}\right) w_{L}\left(r^{\prime}\right)^{1 / 2}}\left[Q_{i}^{a}\left(r^{\prime}\right)-Q_{i}^{a}\left(r_{+}\right)\right. \\
& \left.-C_{i} r_{+} A_{0}^{a \prime}\left(r_{+}\right) w_{L}\left(r_{+}\right)^{1 / 2}+r^{\prime} \tilde{g}_{t i}\left(r^{\prime}\right) A_{0}^{a \prime}\left(r^{\prime}\right)\right] .
\end{aligned}
$$

We still need to determine the constants $C_{i}$. Using (C5), we replace $\tilde{A}_{i}^{a l}$ in $(\mathrm{C} 3)$ and obtain

$$
\begin{aligned}
& {\left[\frac{f^{\prime}(r)}{f(r)}\left(\frac{2}{r}+\frac{w_{T}^{\prime}(r)}{w_{T}(r)}\right)-\frac{8}{3 f(r)}\left(\sum_{a=1}^{n} A_{0}^{a \prime}(r)^{2}+3\right)\right] \tilde{g}_{t i}(r)} \\
& \quad+\left(\frac{1}{r}+\frac{w_{L}^{\prime}(r)}{2 w_{L}(r)}\right) \tilde{g}_{t i}^{\prime}(r)+\tilde{g}_{t i}^{\prime \prime}(r)=\frac{1}{w_{L}(r)^{1 / 2} r f(r)} I(r),
\end{aligned}
$$

where

$$
\begin{aligned}
I(r)= & \sum_{a=1}^{n} 4 A_{0}^{a \prime}(r)\left(Q_{i}^{a}(r)-Q_{i}^{a}\left(r_{+}\right)\right. \\
& \left.-C_{i} r_{+} w_{L}\left(r_{+}\right)^{1 / 2} A_{0}^{a l}\left(r_{+}\right)\right) .
\end{aligned}
$$

A homogeneous solution of this equation $\tilde{g}_{t i}(r)=$ $g_{t t}^{(0)}(r)=f(r)$ can be generated by the infinitesimal coordinate transformation

$$
d t \rightarrow d t-\epsilon(d x+d y+d z), \quad d z \rightarrow d z+\epsilon \frac{d r}{r^{2} w_{L}},
$$

$d x \rightarrow d z+\epsilon \frac{d r}{r^{2} w_{T}}, \quad d y \rightarrow d y+\epsilon \frac{d r}{r^{2} w_{T}}$.

Then, using this homogeneous solution and Appendix D $\left[P(r)=f(r)\right.$ and $E(r)=r w_{L}(r)^{1 / 2}$ there], we bring $(\mathrm{C} 8)$ to the integrable form

$$
\partial_{r}\left(w_{L}(r)^{1 / 2} r f^{2}(r) \partial_{r}\left(\frac{\tilde{g}_{t i}(r)}{f(r)}\right)\right)=I(r) .
$$

Solving this equation for $\tilde{g}_{t i}(r)$ and fixing the integration constants at $r_{+}$, we get

$$
\begin{aligned}
\tilde{g}_{t i}(r)= & f(r) \int_{\infty}^{r} d r^{\prime} \frac{1}{w_{L}\left(r^{\prime}\right)^{1 / 2} r^{\prime}\left(f\left(r^{\prime}\right)\right)^{2}} \\
& \times\left(\int_{r_{+}}^{r^{\prime}} d r^{\prime \prime} I\left(r^{\prime \prime}\right)-w_{L}\left(r_{+}\right)^{1 / 2} r_{+} f^{\prime}\left(r_{+}\right) C_{i}\right) .
\end{aligned}
$$

In the Landau frame we require $u_{\mu} \tau^{\mu \nu}=0$, which in particular implies the absence of corrections to $T^{t i}$. Holographic renormalization [51] translates this into a constraint for the $r^{-2}$ coefficient of $\tilde{g}_{t i}(r)$ which is proportional to the first correction of $T^{t i}$,

$$
\lim _{r \rightarrow \infty} r^{2} \tilde{g}_{t i}(r)=0 .
$$


In the limit $r \rightarrow \infty$, we have the asymptotics

$$
\begin{aligned}
f(r) & =O\left(r^{2}\right), \quad w_{L}(r)=O(1), \\
\int_{r_{+}}^{r} d r^{\prime} I\left(r^{\prime}\right) & =O(1),
\end{aligned}
$$

and, from the vanishing of the $r^{-2}$ coefficient of $\tilde{g}_{t i}(r)$, we obtain the following equation for $C_{i}$ :

$$
w_{L}\left(r_{+}\right)^{1 / 2} r_{+} f^{\prime}\left(r_{+}\right) C_{i}=\int_{r_{+}}^{\infty} d r^{\prime} I\left(r^{\prime}\right) \equiv I_{1}+I_{2} \cdot C_{i}
$$

where we defined the integrals

$$
\begin{aligned}
I_{1} \equiv & 4 \int_{r_{+}}^{\infty} d r^{\prime} \sum_{a=1}^{n} A_{0}^{a \prime}\left(r^{\prime}\right)\left(Q_{i}^{a}\left(r^{\prime}\right)-Q_{i}^{a}\left(r_{+}\right)\right) \\
= & \frac{4}{3} S_{a b c} A_{0}^{a}\left(r_{+}\right) A_{0}^{b}\left(r_{+}\right) A_{0}^{c}\left(r_{+}\right) \epsilon^{i j k}\left(\partial_{j} u_{k}\right) \\
& +2 S_{a b c} A_{0}^{a}\left(r_{+}\right) A_{0}^{b}\left(r_{+}\right) \epsilon^{i j k}\left(\partial_{j} \mathcal{A}_{k}^{c}\right)
\end{aligned}
$$

and

$$
\begin{aligned}
I_{2} & \equiv 4 \int_{r_{+}}^{\infty} d r^{\prime} \sum_{a=1}^{n} A_{0}^{a \prime}\left(r^{\prime}\right)\left(-w_{L}\left(r_{+}\right)^{1 / 2} r_{+} A_{0}^{a \prime}\left(r_{+}\right)\right) \\
& =4 w_{L}\left(r_{+}\right)^{1 / 2} r_{+} \sum_{a=1}^{n} A_{0}^{a}\left(r_{+}\right) A_{0}^{a \prime}\left(r_{+}\right) .
\end{aligned}
$$

Solving this for $C_{i}$, we eventually get

$$
\begin{aligned}
C_{i}= & \frac{4}{r_{+}\left(f^{\prime}\left(r_{+}\right)-4 \sum_{a} A_{0}^{a}\left(r_{+}\right) A_{0}^{a \prime}\left(r_{+}\right)\right)} \cdot \frac{1}{w_{L}\left(r_{+}\right)^{1 / 2}} \\
& \times\left(\frac{1}{3} S_{a b c} A_{0}^{a}\left(r_{+}\right) A_{0}^{b}\left(r_{+}\right) A_{0}^{c}\left(r_{+}\right) \epsilon^{i j k}\left(\partial_{j} u_{k}\right)\right. \\
& \left.+\frac{1}{2} S_{a b c} A_{0}^{a}\left(r_{+}\right) A_{0}^{b}\left(r_{+}\right) \epsilon^{i j k}\left(\partial_{j} \mathcal{A}_{k}^{c}\right)\right) .
\end{aligned}
$$

\section{APPENDIX D: INTEGRABLE FORM OF A LINEAR ORDINARY DIFFERENTIAL EQUATION}

In this appendix we present a method to bring an arbitrary linear ODE of second order to an integrable form. Let us consider a general form of this equation

$$
G\left(g^{\prime \prime}, g^{\prime}, g, r\right) \equiv g^{\prime \prime}(r)+a(r) g^{\prime}(r)+b(r) g(r)=c(r) .
$$

If we know a homogeneous solution $P(r)$ of this equation, i.e.

$$
G\left(P^{\prime \prime}, P^{\prime}, P, r\right)=0,
$$

then we can make the substitution

$$
g(r) \rightarrow P(r) Q(r), \quad Q^{\prime}(r) \rightarrow u(r)
$$

and lower the order of the differential operator (D1)

$$
\begin{aligned}
G & =P(r)\left(u^{\prime}(r)+\left[a(r)+2 \frac{P^{\prime}(r)}{P(r)}\right] u(r)\right) \\
& \equiv P(r)\left(u^{\prime}(r)+F(r) u(r)\right) .
\end{aligned}
$$

The term in the brackets can be represented as

$$
u^{\prime}(r)+F(r) u(r)=\frac{1}{A(r)} \partial_{r}(A(r) u(r))
$$

where

$$
A(r)=\exp \left\{\int F(r) d r\right\}=P(r)^{2} \exp \left\{\int a(r) d r\right\}
$$

Taking into account (D3), we finally bring (D1) to the following integrable form

$$
\frac{1}{P(r) E(r)} \partial_{r}\left(P(r)^{2} E(r) \partial_{r}\left(\frac{g(r)}{P(r)}\right)\right)=c(r)
$$

where we defined

$$
E(r) \equiv \exp \left\{\int a(r) d r\right\}
$$

[1] B. I. Abelev et al. (STAR Collaboration), Phys. Rev. Lett. 103, 251601 (2009); Phys. Rev. C 81, 054908 (2010); D. Gangadharan (STAR Collaboration), J. Phys. G 38, 124166 (2011).

[2] N. N. Ajitanand, S. Esumi, and R. A. Lacey (PHENIX Collaboration), in Proceedings of the RBRC Workshops, Vol. 96, http://quark.phy.bnl.gov/ kharzeev/cpodd/, 2010.

[3] P. Christakoglou, J. Phys. G 38, 124165 (2011).

[4] D. Kharzeev, Phys. Lett. B 633, 260 (2006); D. Kharzeev and A. Zhitnitsky, Nucl. Phys. A797, 67 (2007); D. E. Kharzeev, L. D. McLerran, and H. J. Warringa, Nucl. Phys. A803, 227 (2008).
[5] K. Fukushima, D. E. Kharzeev, and H. J. Warringa, Phys. Rev. D 78, 074033 (2008).

[6] A. Vilenkin, Phys. Rev. D 20, 1807 (1979); 22, 3080 (1980); 22, 3067 (1980).

[7] M. Giovannini and M. E. Shaposhnikov, Phys. Rev. Lett. 80, 22 (1998); 57, 2186 (1998).

[8] A. Y. Alekseev, V. V. Cheianov, and J. Fröhlich, Phys. Rev. Lett. 81, 3503 (1998); A. Vilenkin, Phys. Rev. B 25, 4301 (1982).

[9] P. V. Buividovich, M. N. Chernodub, E. V. Luschevskaya, and M. I. Polikarpov, Phys. Rev. D 80, 054503 (2009); P. V. Buividovich, M. N. Chernodub, D.E. Kharzeev, 
T. Kalaydzhyan, E. V. Luschevskaya, and M. I. Polikarpov, Phys. Rev. Lett. 105, 132001 (2010); V. V. Braguta, P. V. Buividovich, T. Kalaydzhyan, S. V. Kuznetsov, and M. I. Polikarpov, Proc. Sci., LATTICE2010 (2010) 190 [arXiv:1011.3795].

[10] M. Abramczyk, T. Blum, G. Petropoulos, and R. Zhou, Proc. Sci., LAT2009 (2009) 181, http://pos.sissa.it/ archive/conferences/091/181/LAT2009_181.pdf.

[11] A. Yamamoto, Phys. Rev. D 84, 114504 (2011); Phys. Rev. Lett. 107, 031601 (2011).

[12] B. Müller and A. Schäfer, Phys. Rev. C 82, 057902 (2010).

[13] Q. Wang, STAR Presentation given at the conference "Quark Matter 2011," http://drupal.star.bnl.gov/STAR/ presentations/qm2011/quan-wangqm2011/quan-wang.

[14] Q. Wang and P. Surowka, arXiv:1205.4638

[15] A. V. Sadofyev, V. I. Shevchenko, and V. I. Zakharov, Phys. Rev. D 83, 105025 (2011).

[16] A. V. Sadofyev and M. V. Isachenkov, Phys. Lett. B 697, 404 (2011).

[17] S. Pu, J.h. Gao, and Q. Wang, Phys. Rev. D 83, 094017 (2011); J.-H. Gao, Z.-T. Liang, S. Pu, Q. Wang, and X.-N. Wang, arXiv:1203.0725.

[18] Y. Neiman and Y. Oz, J. High Energy Phys. 03 (2011) 023.

[19] M. Lublinsky and I. Zahed, Phys. Lett. B 684, 119 (2010).

[20] T. Kalaydzhyan and I. Kirsch, Phys. Rev. Lett. 106, 211601 (2011).

[21] J. Erdmenger, M. Haack, M. Kaminski, and A. Yarom, J. High Energy Phys. 01 (2009) 055.

[22] R. Ryblewski and W. Florkowski, Phys. Rev. C 77, 064906 (2008).

[23] W. Florkowski, Phys. Lett. B 668, 32 (2008).

[24] R. Ryblewski and W. Florkowski, Eur. Phys. J. C 71, 1761 (2011).

[25] P. F. Kolb, J. Sollfrank, and U. W. Heinz, Phys. Rev. C 62 , 054909 (2000).

[26] K. Behrndt, M. Cvetic, and W. A. Sabra, Nucl. Phys. B553, 317 (1999).

[27] H. U. Yee, J. High Energy Phys. 11 (2009) 085.

[28] A. Gorsky, P. N. Kopnin, and A. V. Zayakin, Phys. Rev. D 83, 014023 (2011).

[29] V. A. Rubakov, arXiv:1005.1888.

[30] A. Gynther, K. Landsteiner, F. Pena-Benitez, and A. Rebhan, J. High Energy Phys. 02 (2011) 110.

[31] A. Rebhan, A. Schmitt, and S. A. Stricker, J. High Energy Phys. 01 (2010) 026.
[32] L. Brits and J. Charbonneau, Phys. Rev. D 83, 126013 (2011).

[33] I. Amado, K. Landsteiner, and F. Pena-Benitez, J. High Energy Phys. 05 (2011) 081.

[34] G. Lifschytz and M. Lippert, Phys. Rev. D 80, 066005 (2009).

[35] C. Hoyos, T. Nishioka, and A. O'Bannon, J. High Energy Phys. 10 (2011) 084.

[36] J. Bhattacharya, S. Bhattacharyya, S. Minwalla, and A. Yarom, arXiv:1105.3733.

[37] Y.-P. Hu, P. Sun, and J.-H. Zhang, Phys. Rev. D 83, 126003 (2011); Y.-P. Hu, arXiv:1112.4227.

[38] R. A. Janik and P. Witaszczyk, J. High Energy Phys. 09 (2008) 026.

[39] M. Chernicoff, D. Fernandez, D. Mateos, and D. Trancanelli, arXiv:1202.3696; D. Mateos and D. Trancanelli, Phys. Rev. Lett. 107, 101601 (2011); J. High Energy Phys. 07 (2011) 054.

[40] J. Erdmenger, P. Kerner, and H. Zeller, J. High Energy Phys. 01 (2012) 059.

[41] S. Bhattacharyya, V.E. Hubeny, S. Minwalla, and M. Rangamani, J. High Energy Phys. 02 (2008) 045.

[42] A. Rebhan and D. Steineder, J. High Energy Phys. 08 (2011) 153.

[43] A. Rebhan and D. Steineder, Phys. Rev. Lett. 108, 021601 (2012).

[44] D. Giataganas, arXiv:1202.4436.

[45] K. Landsteiner, E. Megias, and F. Pena-Benitez, Phys. Rev. Lett. 107, 021601 (2011).

[46] D. E. Kharzeev and D. T. Son, Phys. Rev. Lett. 106, 062301 (2011).

[47] A. Chamblin, R. Emparan, C. V. Johnson, and R. C. Myers, Phys. Rev. D 60, 104026 (1999).

[48] T. Kalaydzhyan and I. Kirsch, J. High Energy Phys. 02 (2011) 053.

[49] S. Kirkpatrick, C.D. Gelatt, and M.P. Vecchi, Science 220, 671 (1983); V. Cerny, Commenius Report No. 82-0540, http://mkweb.bcgsc.ca/papers/cernytravelingsalesman.pdf.

[50] M. Torabian and H. U. Yee, J. High Energy Phys. 08 (2009) 020.

[51] M. Bianchi, D.Z. Freedman, and K. Skenderis, Nucl. Phys. B631, 159 (2002).

[52] B. Sahoo and H. U. Yee, J. High Energy Phys. 11 (2010) 095.

[53] P. Huovinen and P. Petreczky, Nucl. Phys. A837, 26 (2010). 